\title{
Selection of Tightened-Normal-Tightened sampling scheme under the implications of intervened Poisson distribution
}

\author{
S. Azarudheen \\ Assistant Professor, Department of Statistics, \\ CHRIST, India \\ sazaru1990@gmail.com \\ K. Pradeepa Veerakumari \\ Assistant Professor, Department of Statistics, \\ Bharathiar University, India \\ sadeep_13@yahoo.co.in
}

\begin{abstract}
Tightened-normal-tightened (TNT) sampling scheme is one of the most frequently used sampling schemes for making decisions about the finished product lots by examining certain samples from the lots. TNT sampling scheme includes two attribute sampling plans, one for tightened inspection and other for normal inspection along with switching rules. This paper introduces a procedure for TNT by incorporating two single sampling plans (SSP) under the conditions of intervened Poisson distribution (IPD) for the lots which may have a possibility of some intervention during the production process. The paper also assesses the performance of the proposed scheme procedure through its operating characteristic curves. Also, the unity value table is provided for certain parameters of specified producer's risk and consumer's risk for shop floor conditions. Further, the efficiency of proposed TNT scheme over the individual SSP under the conditions of IPD is demonstrated with illustrations.
\end{abstract}

Key words: Sampling plan, Sampling scheme, Tightened-normal-tightened sampling scheme, single sampling plan, intervened Poisson distribution.

\section{Introduction}

Quality control (QC) is a set of procedures used by organizations to achieve the quality parameters or quality goals and continuously improve the organization's ability to ensure, that the products will meet the quality goals. The statistical methods used to mentor and maintain the quality of products and services are referred as Statistical Quality Control (SQC). Acceptance sampling (AS) is one of the major areas of SQC, which is supportive to sentence about the quality of the lots through certain procedures. According to Dodge (1969) AS is characterized into four categories such as lot-by-lot attribute sampling plans, lot-by-lot variable sampling plans, continuous sampling plans and special purpose sampling plan. Sampling plans are the set of rules which is to be followed for sentencing any lot about its quality. As described by Schilling and Neubauer (2009) a sampling scheme involves procedure for the use of a set of specific plans, a sampling system is viewed as an assigned grouping of two or three sampling plans and the rules for using these plans for sentencing the lots.

Calvin (1977) introduced a Tightened-Normal-Tightened sampling scheme which involved two single sampling plans with two samples sizes say $\mathrm{n} 1$ and $\mathrm{n} 2$ and acceptance number $\mathrm{c}=0$ and designated as TNT $-(\mathrm{n} 1, \mathrm{n} 2,0)$. According to Schilling (1982), the TNT $(\mathrm{n} 1, \mathrm{n} 2,0)$ scheme is desirable for compliance testing since nonconforming units in the sample are not allowed in compliance testing. The operating procedure of the scheme is based on the 
switching rules of MIL-STD-105D (1963) involving only normal and tightened inspection which as proposed by Dodge (1965). Soundararajan and Vijaaraghavan (1990) extended the work of Calvin (1977) on TNT schemes and developed a table of parametric values of various TNT schemes which permit the convenience of designing one's own scheme. They described procedures for designing the schemes for given quality levels such as AQL, LQL and AOQL. Further, Soundararajan and Vijayaraghavan (1992) revealed that the TNT scheme with s $=4$ and

$\mathrm{t}=5$ provides a discriminating OC curve than the schemes based on other sets of values of $\mathrm{s}$ and t. Vijayaraghavan and Soundararajan (1996) proposed a TNT sampling scheme which incorporates two single sampling plans of same sample size and two different acceptance numbers along with certain switching rules. This scheme was designated as TNT - (n, c1, c2). The tightened and normal inspection plans are specified by the parameters (n, c1) and (n, c2) respectively, where $\mathrm{c} 1<\mathrm{c} 2$. Subramani and Haridoss (2012) constructed TNT sampling scheme under the conditions of weighted Poisson distribution indexed through AQL and LQL. Vijayaraghavan et al. (2014) presented an iterative procedure for selection of TNT - (n, c1, c2) sampling schemes with minimum sample size for two specified points on the OC curve namely AQL and LQL.

However, when some intervention is applied to the production process with an intention to improve the quality of the products during the observational period, then the mean of the rare event $\mathrm{p}$ may get altered. In such situation the appropriate probability distribution for number of defectives in the sample is modeled by Intervened Poisson distribution (IPD) proposed by Shanmugam (1985). IPD has a more real time applications in the fields such as agriculture, epidemiology, public health, process control, medicine etc., which can be found in Shanmugam (1985, 2001) and Huang and Fung (1989). Pradeepa Veerakumari and Azarudheen (2016) devised a single sampling plan for the above situations by imparting the conditions of IPD and proved that the consumer's risk and producer's risk reduces while comparing with the conventional single sampling plan proposed by Cameron (1952).

This paper formulates the idea of designing a TNT sampling scheme by incorporating two single sampling plans under the conditions of IPD. A detailed genesis of IPD is given in section 2. Section 3 provides an operating procedure for TNT sampling scheme. In section 4 a designing procedure of TNT sampling scheme under the conditions of IPD is given. Section 5 provides a selection procedure for obtaining the optimum sampling scheme for the proposed scheme. Numerical illustrations are provided to describe the effectiveness of TNT scheme over individual single sampling plans under the conditions of IPD.

\section{A Genesis and properties of IPD}

Let $X_{1}$ be the number of defectives in a lot. In practice, the lot formed from a process can never be perfect due to random fluctuations which imply the event $X_{1}>0$. Since the random variable $X_{l}$ is a rare event, the appropriate distribution which can describe the random variable is zero-truncated Poisson distribution with probability function

$$
P\left(X_{1}=x_{1}\right)=\frac{\theta^{x_{1}}}{\left(e^{\theta}-1\right) x_{1} !} ; x_{1}=1,2, \ldots
$$


where $\theta>0$ is called incidence parameter. In this example, if the production engineer makes any changes in a production system in order to improve the quality, then the incidence parameter can change from $\theta$ to another parameter $\rho \theta$, where $\rho \geq 0$ is called an intervention parameter and let $X_{2}$ be the number of defectives that occurred after making changes in the production process. It is noted that $X_{2}$ is a Poisson random variable with mean $\rho \theta$.

Assuming that our record has only a random variable $X=X_{1}+X_{2}$ that is, the total number of defectives occurred all together, then the random variable $X$ is modeled by IPD with probability function,

$$
P(X=x)=\frac{\left[(1+\rho)^{x}-\rho^{x}\right] \theta^{x}}{e^{\rho \vartheta}\left(e^{\theta}-1\right) x !} ; x=1,2, \ldots
$$

The mean and variance of the $\operatorname{IPD}(\theta, \rho)$ are

$$
\mu=E(X)=\theta\left[(1+\rho)+\frac{1}{\left(e^{\theta}-1\right)}\right]
$$

and

$$
\sigma^{2}=\operatorname{Var}(X)=\mu-e^{\theta}\left(\frac{\theta}{e^{\theta}-1}\right)^{2}
$$

respectively. It is noticed from equations (3) and (4) the variance is less than its mean. When the intervention parameter $\rho$ becomes zero, IPD reduces to zero truncated Poisson distribution. The higher order moments of IPD are obtained by differentiating the moment generating function

$$
\phi_{x}(u)=\frac{e^{\rho \theta\left(e^{u}-1\right)}\left(e^{\theta e^{u}}-1\right)}{e^{\theta}-1}
$$

The additional properties of IPD are given by Shanmugam (1985).

\section{Tightened-Normal-Tightened sampling schemes of type TNT $-\left(n, c_{1}, c_{2}\right)$}

Tightened-Normal-Tightened sampling scheme of type TNT $-\left(n, c_{1}, c_{2}\right)$, is a sampling scheme incorporating two single sampling plans of same sample size, but two different acceptance numbers together with the switching rules to switch between the plans. The tightened and normal inspection plans are specified by the parameters $\left(n, c_{1}\right)$ and $\left(n, c_{2}\right)$ respectively, where $c_{1}<c_{2}$. The TNT scheme also has additional two parameters viz., $s$ and $t$, to represent the criteria for switching to tightened inspection and to normal inspection respectively. The operating procedure for TNT $-\left(n, c_{1}, c_{2}\right)$ scheme is as follows,

i. Start with tightened inspection, with the sample size $n$ and the acceptance number $c_{1}$.

ii. When $t$ lots are accepted in tightened inspection switch to normal inspection with the same sample size $n$ and the acceptance number $c_{2}$, where $c_{1}<c_{2}$.

iii. When as additional lot is rejected in the next $s$ lots after a rejection, switch to tightened inspection.

According to Vijayaraghavan and Soundararajan (1996), the OC function of the scheme is given as, 


$$
P_{a}(p)=\frac{P_{1}\left(1-P_{2}^{s}\right)\left(1-P_{1}^{t}\right)\left(1-P_{2}\right)+P_{2} P_{1}^{t}\left(1-P_{1}\right)\left(2-P_{2}^{s}\right)}{\left(1-P_{2}^{s}\right)\left(1-P_{1}^{t}\right)\left(1-P_{2}\right)+P_{1}^{t}\left(1-P_{1}\right)\left(2-P_{2}^{s}\right)}
$$

where $P_{1}$ is the probability of accepting the lot under the tightened inspection and $P_{2}$ is the probability of accepting the lot under the normal inspection. According to Schilling (1982), the TNT schemes matches the normal - tightened plans of MIL-STD-105D (1963) when the switching criteria are set at $s=4$ and $t=5$. Soundararajan and Vijayaraghavan (1992) observed that the OC curve of a TNT scheme has a desirable shape for the case where $s=4$ and $t=5$. Thus, the design and evaluation of TNT schemes in this sections are presented for the case $s=4$ and $t=5$.

\section{Designing of TNT - $\left(n, c_{1}, c_{2}\right)$ with SSP under the conditions of IPD as reference plan}

According to Schilling (1982), the TNT schemes are more popularly used sampling schemes in compliance sampling. Calvin (1977) proposed TNT sampling plan using two single sampling plans with two different sample sizes $n_{1}$ and $n_{2}$ and zero acceptance number. Further, Vijayaraghavan and Soundararajan (1996) developed TNT sampling plan using two single sampling plans with one sample size $\mathrm{n}$ and two acceptance numbers $c_{1}$ and $c_{2}$. Suppose the production engineers makes some changes during the production process in order to improve the quality of the products and if the lots are formed by the products produced before and after the intervention took place. If a sequence of such lots are submitted for quality check then to address the prevalence of such intervention in the lots TNT under the conditions of intervened Poisson distribution is proposed by considering single sampling plan as reference plan.

The TNT with SSP under the conditions of IPD as reference plan is operated as dictated in section 3 by combining the operating procedure of single sampling plan under the conditions of IPD sentence the individual lots. SSP under the conditions of IPD is portrayed by sample size $n$, acceptance number $c$ and intervention parameter $\rho$. Its operating procedure is as follows,

1. Take a random sample of $n$ units from a lot of size $N$ units.

2. Count the number of defective units, $X=x$ in the sample.

3. If $x \leq c$, accept the lot; otherwise reject the lot.

The OC function of SSP is defined as,

$$
P_{a}(p)=P[x \leq c]
$$

where $p$ symbolizes the lot proportion defectives. In practice, the values of $P_{a}(p)$ can be determined for different values of $p$ using different probability models. As quoted by Schilling and Neubauer (2009), when $\mathrm{n}$ is large, $p$ is small and $n / N \leq 0.10$ such that $n p<5$, the proportion defective $p$ is distributed according to the Poisson distribution with mean $n p$.

When some intervention is applied to the production process with an intention to improve the quality, of the products during the observational period, then the mean of the rare event $p$ may get altered. In such situation the appropriate probability distribution for number of defectives in the sample is modeled by IPD.

The OC function for SSP under the conditions of $\operatorname{IPD}(\theta, \rho)$ can be defined as,

$$
P_{a}(p)=\sum_{x=1}^{c} P(X=x \mid \theta, \rho)
$$




$$
P_{a}(p)=\sum_{x=1}^{c} P(X=x \mid \theta, \rho)=\sum_{x=1}^{c} \frac{\left[(1+\rho)^{x}-\rho^{x}\right] \theta^{x}}{e^{\rho \theta}\left(e^{\theta}-1\right) x !}
$$

where $\theta=n p$ and $\rho$ is the intervention parameter which is measured in percentage. Hence, by obtaining an estimated value for $\rho$, the probability of acceptance can be calculated using equation (9) for given values of $n, c$ and $p$.

This implies that TNT with SSP under the conditions of IPD as reference plan is specified by following parameters,

i. SSP under the conditions of IPD parameters $n, c$ and $\rho$,

ii. Tightened and normal probabilities of acceptance $P_{1}$ and $P_{2}$ respectively,

iii. Tightened and normal acceptance numbers say $c_{1}$ and $c_{2}$ respectively.

The OC function of TNT with SSP under the conditions of IPD as reference plan is defined as given in equation (6) where $P_{1}$ and $P_{2}$ are probabilities of acceptance of the lots under the tightened and normal inspections which is determined by equation (9) for their corresponding acceptance numbers $c_{1}$ and $c_{2}$.

\section{Determination of optimal parameters for $T N T-\left(n, c_{1}, c_{2}\right)$ with SSP under the conditions of IPD as reference plan}

The prime objective of designing TNT with SSP as reference plan under the conditions of IPD is to obtain an optimum combination of parameters $\left(n, c_{1}, c_{2}\right)$ so that the scheme can protect both consumer as well as producer. This can be achieved by specifying $\left(p_{1}, \alpha, p_{2}, \beta\right)$ and applying the conditions $P_{a}\left(p_{1}\right)=1-\alpha$ and $P_{a}\left(p_{2}\right)=\beta$. Since, the parameters $n, c_{1}$ and $c_{2}$ are integer random variables, so the above conditions may not be true for all the situations. Hence, these conditions may be modified as $P_{a}\left(p_{1}\right) \geq 1-\alpha$ and $P_{a}\left(p_{2}\right) \leq \beta$. Thus, by following these conditions the unity values for the proposed plan are computed and tabulated in Table 1. Further, the values of operating ratio (OR), a measure of discrimination, are also computed and tabulated in Table 2.

The parameters of proposed sampling schemes using the Tables 1 and 2 can be obtained by applying a search procedure as follows,

i. For the estimated values of $\rho$ and specified values of $p_{1}, \alpha, p_{2}, \beta$, also specify $s$ and $t$ values and compute the operating ratio $O R=p_{2} / p_{1}$.

ii. Select $O R$ from the Table 2 in the column headed $O R$ that is nearly equal to the computed ratio $O R$ value corresponding to specified $\alpha$ and $\beta$. 
Table 1 Unity values for TNT - $\left(n, c_{1}, c_{2}\right)$ sampling scheme with SSP under the conditions of IPD as reference plan for $s=4$ and $t=5$

\begin{tabular}{|c|c|c|c|c|c|c|c|c|c|}
\hline$\rho$ & $c_{1}$ & $c_{2}$ & 0.99 & 0.95 & 0.75 & 0.5 & 0.1 & 0.05 & 0.01 \\
\hline \multirow{10}{*}{0.01} & 1 & 2 & 0.2492 & 0.5099 & 0.9239 & 1.3675 & 3.5684 & 4.4586 & 6.3429 \\
\hline & 1 & 3 & 0.6333 & 0.9330 & 1.2806 & 1.5886 & 3.5686 & 4.4582 & 6.3685 \\
\hline & 2 & 3 & 0.6599 & 1.1432 & 1.8809 & 2.5786 & 5.2094 & 6.1787 & 8.2159 \\
\hline & 1 & 4 & 1.0282 & 1.3201 & 1.6210 & 1.8610 & 3.5689 & 4.4584 & 6.3573 \\
\hline & 2 & 4 & 1.1284 & 1.6590 & 2.2426 & 2.7477 & 5.2095 & 6.1786 & 8.1829 \\
\hline & 3 & 4 & 1.1284 & 1.8061 & 2.7790 & 3.6562 & 6.5995 & 7.6571 & 9.9107 \\
\hline & 1 & 5 & 1.4388 & 1.6756 & 1.9492 & 2.1525 & 3.5701 & 4.4598 & 6.2943 \\
\hline & 2 & 5 & 1.6564 & 2.1195 & 2.5928 & 2.9755 & 5.2098 & 6.1790 & 8.2698 \\
\hline & 3 & 5 & 1.6564 & 2.3640 & 3.1408 & 3.7957 & 6.5996 & 7.6570 & 9.8663 \\
\hline & 4 & 5 & 1.6564 & 2.4845 & 3.6545 & 4.6835 & 7.9104 & 9.0503 & 11.4215 \\
\hline \multirow{10}{*}{0.05} & 1 & 2 & 0.2426 & 0.4813 & 0.8705 & 1.2875 & 3.3888 & 4.2441 & 6.0633 \\
\hline & 1 & 3 & 0.6056 & 0.8848 & 1.2125 & 1.5024 & 3.3889 & 4.2438 & 6.0828 \\
\hline & 2 & 3 & 0.6261 & 1.0900 & 1.7949 & 2.4627 & 4.9959 & 5.9298 & 7.8900 \\
\hline & 1 & 4 & 0.9940 & 1.2547 & 1.5394 & 1.7660 & 3.3893 & 4.2440 & 6.0714 \\
\hline & 2 & 4 & 1.0726 & 1.5862 & 2.1441 & 2.6273 & 4.9959 & 5.9296 & 7.8577 \\
\hline & 3 & 4 & 1.1284 & 1.7293 & 2.6638 & 3.5075 & 6.3428 & 7.3612 & 9.5367 \\
\hline & 1 & 5 & 1.3547 & 1.5950 & 1.8546 & 2.0472 & 3.3905 & 4.2453 & 6.0066 \\
\hline & 2 & 5 & 1.5584 & 2.0290 & 2.4819 & 2.8490 & 4.9962 & 5.9300 & 7.9460 \\
\hline & 3 & 5 & 1.5793 & 2.2670 & 3.0131 & 3.6425 & 6.3428 & 7.3610 & 9.4854 \\
\hline & 4 & 5 & 1.5947 & 2.3838 & 3.5097 & 4.5003 & 7.6072 & 8.7041 & 10.9864 \\
\hline \multirow{10}{*}{0.1} & 1 & 2 & 0.2188 & 0.4493 & 0.8111 & 1.1988 & 3.1858 & 4.0011 & 5.7304 \\
\hline & 1 & 3 & 0.5679 & 0.8306 & 1.1362 & 1.4060 & 3.1861 & 4.0161 & 5.7563 \\
\hline & 2 & 3 & 0.5952 & 1.0292 & 1.6962 & 2.3326 & 4.7489 & 5.6419 & 7.5160 \\
\hline & 1 & 4 & 0.9205 & 1.1809 & 1.4475 & 1.6591 & 3.1866 & 4.0204 & 5.7440 \\
\hline & 2 & 4 & 1.0045 & 1.5025 & 2.0308 & 2.4885 & 4.7490 & 5.6417 & 7.4813 \\
\hline & 3 & 4 & 1.0223 & 1.6411 & 2.5312 & 3.3360 & 6.0468 & 7.0202 & 9.0912 \\
\hline & 1 & 5 & 1.2955 & 1.5039 & 1.7477 & 1.9283 & 3.1876 & 4.0018 & 5.6887 \\
\hline & 2 & 5 & 1.4956 & 1.9251 & 2.3545 & 2.7001 & 4.7492 & 5.6422 & 7.5676 \\
\hline & 3 & 5 & 1.5199 & 2.1555 & 2.8660 & 3.4659 & 6.0469 & 7.0201 & 9.0527 \\
\hline & 4 & 5 & 1.5308 & 2.2681 & 3.3429 & 4.2894 & 7.2585 & 8.3060 & 10.4887 \\
\hline
\end{tabular}


Table 1 (Continued)

\begin{tabular}{|c|c|c|c|c|c|c|c|c|c|}
\hline$\rho$ & $c_{1}$ & $c_{2}$ & 0.99 & 0.95 & 0.75 & 0.5 & 0.1 & 0.05 & 0.01 \\
\hline \multirow{10}{*}{0.15} & 1 & 2 & 0.2066 & 0.4209 & 0.7588 & 1.1208 & 3.0039 & 3.7978 & 5.4368 \\
\hline & 1 & 3 & 0.5333 & 0.7820 & 1.0682 & 1.3205 & 3.0037 & 3.7677 & 5.4604 \\
\hline & 2 & 3 & 0.5609 & 0.9739 & 1.6063 & 2.2107 & 4.5220 & 5.3774 & 7.1707 \\
\hline & 1 & 4 & 0.8960 & 1.1146 & 1.3652 & 1.5635 & 3.0040 & 3.7610 & 5.4507 \\
\hline & 2 & 4 & 0.9672 & 1.4263 & 1.9273 & 2.3617 & 4.5221 & 5.3773 & 7.1416 \\
\hline & 3 & 4 & 0.9800 & 1.5605 & 2.4096 & 3.1787 & 5.7753 & 6.7076 & 8.6910 \\
\hline & 1 & 5 & 1.2220 & 1.4220 & 1.6510 & 1.8216 & 3.0055 & 3.7825 & 5.3949 \\
\hline & 2 & 5 & 1.4297 & 1.8301 & 2.2377 & 2.5677 & 4.5223 & 5.3777 & 7.2210 \\
\hline & 3 & 5 & 1.4374 & 2.0535 & 2.7313 & 3.3038 & 5.7753 & 6.7074 & 8.6510 \\
\hline & 4 & 5 & 1.4503 & 2.1622 & 3.1902 & 4.0961 & 6.9394 & 7.9423 & 10.0271 \\
\hline \multirow{10}{*}{0.2} & 1 & 2 & 0.1963 & 0.3955 & 0.7123 & 1.0517 & 2.8394 & 3.5676 & 5.1704 \\
\hline & 1 & 3 & 0.5102 & 0.7383 & 1.0074 & 1.2441 & 2.8397 & 3.5829 & 5.1926 \\
\hline & 2 & 3 & 0.5381 & 0.9236 & 1.5241 & 2.0992 & 4.3132 & 5.1339 & 6.8555 \\
\hline & 1 & 4 & 0.8262 & 1.0549 & 1.2912 & 1.4777 & 2.8399 & 3.5736 & 5.1792 \\
\hline & 2 & 4 & 0.9268 & 1.3565 & 1.8326 & 2.2455 & 4.3133 & 5.1338 & 6.8277 \\
\hline & 3 & 4 & 0.9382 & 1.4866 & 2.2979 & 3.0338 & 5.5252 & 6.4198 & 8.3226 \\
\hline & 1 & 5 & 1.1491 & 1.3480 & 1.5645 & 1.7254 & 2.8414 & 3.5841 & 5.1257 \\
\hline & 2 & 5 & 1.3660 & 1.7431 & 2.1308 & 2.4448 & 4.3154 & 5.1587 & 6.9023 \\
\hline & 3 & 5 & 1.3629 & 1.9598 & 2.6074 & 3.1546 & 5.5253 & 6.4197 & 8.2818 \\
\hline & 4 & 5 & 1.3776 & 2.0650 & 3.0497 & 3.9183 & 6.6461 & 7.6081 & 9.6035 \\
\hline
\end{tabular}


Table 2 Operating Ratio values for $T N T-\left(n, c_{1}, c_{2}\right)$ sampling scheme with SSP under the conditions of IPD as reference plan for $s=4$ and $t=5$

\begin{tabular}{|c|c|c|c|c|c|c|c|c|}
\hline$\rho$ & $c_{1}$ & $c_{2}$ & $\begin{array}{l}\alpha=0.05 \\
\beta=0.10\end{array}$ & $\begin{array}{l}\alpha=0.05 \\
\beta=0.05\end{array}$ & $\begin{array}{l}\alpha=0.05 \\
\beta=0.01\end{array}$ & $\begin{array}{l}\alpha=0.01 \\
\beta=0.10\end{array}$ & $\begin{array}{l}\alpha=0.01 \\
\beta=0.05\end{array}$ & $\begin{array}{l}\alpha=0.01 \\
\beta=0.01\end{array}$ \\
\hline \multirow{10}{*}{0.01} & 1 & 2 & 6.998 & 12.440 & 12.440 & 14.320 & 17.892 & 25.453 \\
\hline & 1 & 3 & 3.825 & 6.826 & 6.826 & 5.635 & 7.040 & 10.056 \\
\hline & 2 & 3 & 4.557 & 7.187 & 7.187 & 7.894 & 9.363 & 12.450 \\
\hline & 1 & 4 & 2.704 & 4.816 & 4.816 & 3.471 & 4.336 & 6.183 \\
\hline & 2 & 4 & 3.140 & 4.932 & 4.932 & 4.617 & 5.475 & 7.252 \\
\hline & 3 & 4 & 3.654 & 5.487 & 5.487 & 5.848 & 6.786 & 8.783 \\
\hline & 1 & 5 & 2.131 & 3.756 & 3.756 & 2.481 & 3.100 & 4.375 \\
\hline & 2 & 5 & 2.458 & 3.902 & 3.902 & 3.145 & 3.730 & 4.993 \\
\hline & 3 & 5 & 2.792 & 4.174 & 4.174 & 3.984 & 4.623 & 5.957 \\
\hline & 4 & 5 & 3.184 & 4.597 & 4.597 & 4.776 & 5.464 & 6.896 \\
\hline \multirow{10}{*}{0.05} & 1 & 2 & 7.041 & 12.598 & 12.598 & 13.971 & 17.497 & 24.997 \\
\hline & 1 & 3 & 3.830 & 6.875 & 6.875 & 5.596 & 7.007 & 10.043 \\
\hline & 2 & 3 & 4.583 & 7.239 & 7.239 & 7.980 & 9.471 & 12.603 \\
\hline & 1 & 4 & 2.701 & 4.839 & 4.839 & 3.410 & 4.270 & 6.108 \\
\hline & 2 & 4 & 3.150 & 4.954 & 4.954 & 4.658 & 5.528 & 7.326 \\
\hline & 3 & 4 & 3.668 & 5.515 & 5.515 & 5.621 & 6.523 & 8.451 \\
\hline & 1 & 5 & 2.126 & 3.766 & 3.766 & 2.503 & 3.134 & 4.434 \\
\hline & 2 & 5 & 2.462 & 3.916 & 3.916 & 3.206 & 3.805 & 5.099 \\
\hline & 3 & 5 & 2.798 & 4.184 & 4.184 & 4.016 & 4.661 & 6.006 \\
\hline & 4 & 5 & 3.191 & 4.609 & 4.609 & 4.770 & 5.458 & 6.889 \\
\hline \multirow{10}{*}{0.1} & 1 & 2 & 7.091 & 12.754 & 12.754 & 14.563 & 18.290 & 26.195 \\
\hline & 1 & 3 & 3.836 & 6.931 & 6.931 & 5.610 & 7.071 & 10.135 \\
\hline & 2 & 3 & 4.614 & 7.303 & 7.303 & 7.979 & 9.479 & 12.628 \\
\hline & 1 & 4 & 2.698 & 4.864 & 4.864 & 3.462 & 4.368 & 6.240 \\
\hline & 2 & 4 & 3.161 & 4.979 & 4.979 & 4.728 & 5.616 & 7.448 \\
\hline & 3 & 4 & 3.685 & 5.540 & 5.540 & 5.915 & 6.867 & 8.893 \\
\hline & 1 & 5 & 2.119 & 3.783 & 3.783 & 2.460 & 3.089 & 4.391 \\
\hline & 2 & 5 & 2.467 & 3.931 & 3.931 & 3.175 & 3.772 & 5.060 \\
\hline & 3 & 5 & 2.805 & 4.200 & 4.200 & 3.979 & 4.619 & 5.956 \\
\hline & 4 & 5 & 3.200 & 4.624 & 4.624 & 4.742 & 5.426 & 6.852 \\
\hline
\end{tabular}


Table 2 (Continued)

\begin{tabular}{|c|c|c|c|c|c|c|c|c|}
\hline$\rho$ & $c_{1}$ & $c_{2}$ & $\begin{array}{l}\alpha=0.05 \\
\beta=0.10\end{array}$ & $\begin{array}{l}\alpha=0.05 \\
\beta=0.05\end{array}$ & $\begin{array}{l}\alpha=0.05 \\
\beta=0.01\end{array}$ & $\begin{array}{l}\alpha=0.01 \\
\beta=0.10\end{array}$ & $\begin{array}{l}\alpha=0.01 \\
\beta=0.05\end{array}$ & $\begin{array}{l}\alpha=0.01 \\
\beta=0.01\end{array}$ \\
\hline \multirow{10}{*}{0.15} & 1 & 2 & 7.137 & 12.918 & 12.918 & 14.541 & 18.383 & 26.318 \\
\hline & 1 & 3 & 3.841 & 6.983 & 6.983 & 5.633 & 7.065 & 10.240 \\
\hline & 2 & 3 & 4.643 & 7.363 & 7.363 & 8.061 & 9.586 & 12.783 \\
\hline & 1 & 4 & 2.695 & 4.890 & 4.890 & 3.353 & 4.198 & 6.083 \\
\hline & 2 & 4 & 3.171 & 5.007 & 5.007 & 4.675 & 5.559 & 7.384 \\
\hline & 3 & 4 & 3.701 & 5.570 & 5.570 & 5.893 & 6.845 & 8.869 \\
\hline & 1 & 5 & 2.114 & 3.794 & 3.794 & 2.459 & 3.095 & 4.415 \\
\hline & 2 & 5 & 2.471 & 3.946 & 3.946 & 3.163 & 3.761 & 5.051 \\
\hline & 3 & 5 & 2.812 & 4.213 & 4.213 & 4.018 & 4.666 & 6.019 \\
\hline & 4 & 5 & 3.209 & 4.637 & 4.637 & 4.785 & 5.476 & 6.914 \\
\hline \multirow{10}{*}{0.2} & 1 & 2 & 7.179 & 13.072 & 13.072 & 14.464 & 18.174 & 26.339 \\
\hline & 1 & 3 & 3.846 & 7.033 & 7.033 & 5.566 & 7.023 & 10.178 \\
\hline & 2 & 3 & 4.670 & 7.423 & 7.423 & 8.015 & 9.541 & 12.740 \\
\hline & 1 & 4 & 2.692 & 4.910 & 4.910 & 3.437 & 4.325 & 6.268 \\
\hline & 2 & 4 & 3.180 & 5.033 & 5.033 & 4.654 & 5.539 & 7.367 \\
\hline & 3 & 4 & 3.717 & 5.599 & 5.599 & 5.889 & 6.843 & 8.871 \\
\hline & 1 & 5 & 2.108 & 3.802 & 3.802 & 2.473 & 3.119 & 4.461 \\
\hline & 2 & 5 & 2.476 & 3.960 & 3.960 & 3.159 & 3.777 & 5.053 \\
\hline & 3 & 5 & 2.819 & 4.226 & 4.226 & 4.054 & 4.710 & 6.077 \\
\hline & 4 & 5 & 3.218 & 4.651 & 4.651 & 4.824 & 5.523 & 6.971 \\
\hline
\end{tabular}

iii. Determine the $c_{1}$ and $c_{2}$ values corresponding to the ratio $O R$ value.

iv. Locate $c_{1}$ and $c_{2}$ values in Table 1 and select the corresponding $n p_{1}$ and $n p_{2}$ values.

v. The sample size $n$ is then calculated by dividing $n p_{1}$ by $p_{1}$ or $n p_{2}$ by $p_{2}$ and choose whichever is larger.

The above procedure is described in the following illustration.

\section{Illustration 1}

Consider the strength $\left(p_{1}, \alpha, p_{2}, \beta\right)$ to be $0.02,0.05,0.15$ and 0.10 respectively and the estimated value of the intervention parameter $\rho=10 \%$ along with $s=4$ and $t=5$. To determine the plan parameters corresponding to the specified strengths proceed as follows, first compute the operating ratio $O R=p_{2} / p_{1}=0.15 / 0.02=7.5$. Enter the column headed with $\alpha=0.05$ and $\beta=0.10$ in Table 2 with $\rho=0.1$ and an $O R$ is chosen which is near to computed $O R$ as 7.091 and its associated $c_{1}$ and $c_{2}$ values are chosen as 1 and 2 respectively. Now enter the Table 1 with the values $\rho=0.1, c_{1}=1, \mathrm{c}_{2}=2,1-\alpha=0.95$ and $\beta=0.10$ and select $n p_{1}$ value and $n p_{2}$ values as 0.4493 and 3.1858 respectively. At $p_{1}=0.02$ the $n$ value is determined 
as $n p_{1} / p_{1}=22.46 \approx 23$ and at $p_{2}=0.15$ the $n$ value is determined as $n p_{2} / p_{2}=21.23 \approx 22$. Therefore the number of products to be inspected is 23 in each lot. Thus, the optimum TNT with SSP under the conditions of IPD as reference plan for the given specifications is $(23,1,2)$.

\section{Implementation of TNT - $\left(n, c_{1}, c_{2}\right)$ with SSP under the conditions of IPD}

The optimum TNT plan obtained in illustration 1 can be implemented as follows,

1. Start with tightened inspection with SSP under the conditions of IPD by inspecting every lot submitted in order with sample size $n=23$ and acceptance number 1 .

2. If 5 lots are accepted during tightened inspection, then switch to normal inspection. During normal inspection, inspect the lots using SSP under the conditions of IPD with the sample size $n=23$ and acceptance number 2 .

3. If a lot is rejected during normal inspection then switch to tightened inspection and repeat step 1 .

\section{A comparative study of proposed sampling scheme over SSP under the conditions of IPD}

In this section, the TNT $-\left(n, c_{1}, c_{2}\right)$ with SSP under the conditions of IPD as reference plan is compared with its reference plans, such as normal and tightened SSP under the conditions of IPD. The general comparison of any sampling plans is done by comparing its OC curves and OC function values for the same parameter values. Figure 1 displays the OC curves for the parameters of proposed system with parameters, $n=$ $100, c_{1}=1$ and $c_{2}=3$, the tightened SSP under the conditions of $\operatorname{IPD}(100,1)$ and normal SSP under the conditions of $\operatorname{IPD}(100,3)$ with $\rho=0.01$. From the Figure 1 , it is observed that the OC curve of TNT $-\left(n, c_{1}, c_{2}\right)$ with SSP under the conditions of IPD is bounded by its reference plans. Also, it is noted that the proposed sampling system coincides with that of normal single sampling plan under the conditions of IPD for the lots having low proportion defectives. Conversely, the OC curve of TNT $-\left(n, c_{1}, c_{2}\right)$ with SSP under the conditions of IPD approaches tightened SSP under the conditions of IPD when the submitted lots quality is reduced. Thus, the TNT $-\left(n, c_{1}, c_{2}\right)$ with SSP under the conditions of IPD achieves combined advantages of both component plans by providing protection to both the consumer and producer.

Any sampling plan should reduce the sample size in order to reduce the inspection cost. Hence, the proposed sampling system is compared with SSP under the conditions of IPD in terms of sample size required for inspection. To achieve this, the operating ratio is used to discriminate the plans. Suppose, the intervention parameter is estimated as $\rho=0.15$ and the plan is to be designed for the strengths, $p_{1}=0.009, \alpha=0.05, p_{2}=0.03$ and $\beta=$ 0.10 then the operating ratio $(\mathrm{OR})$ is calculated to find the parameters. Here, the $O R=p_{2} / p_{1}=$ $0.03 / 0.009=3.33$. From Table 2 the nearest OR value to the calculated OR is 3.209 and corresponding $c_{1}$ and $c_{2}$ values are 4 and 5 respectively. Thus, the $n p_{1}$ value was found to be 2.1622 from the Table 1. Hence, the sample size is computed as $n=n p_{1} / p_{1}=2.1622 / 0.009=$ $240.24 \approx 241$.

From the Tables proposed by Pradeepa Veerakamari and Azarudheen (2017), the nearest OR value for the computed OR value is found to be 3.2640 and the corresponding to acceptance number value is 6 . Further, the unity value $n p_{1}$ corresponding the acceptance number 6 and intervention parameter 0.15 is 2.8051 . 


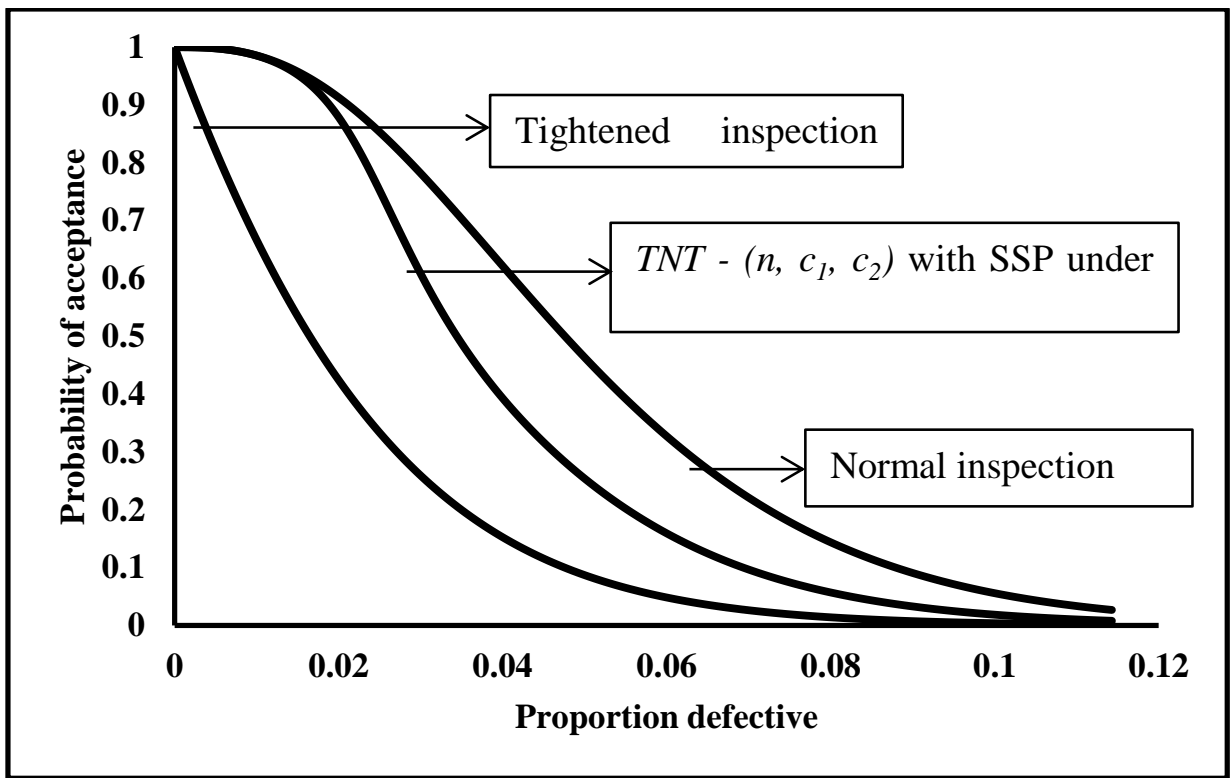

Figure 1. OC curves of $T N T-\left(n, c_{1}, c_{2}\right)$ with SSP under the conditions of IPD and SSP under the conditions of IPD plan under tightened and normal inspection

Therefore, the sample size of SSP under the conditions of IPD for the given specifications is computed as, $n=n p_{1} / p_{1}=2.8051 / 0.009=311.67 \approx 312$. As a result, there is $22.75 \%$ of reduction in sample size of proposed TNT $-\left(n, c_{1}, c_{2}\right)$ when compared with SSP under the conditions of IPD for the specified conditions. Thus, the TNT $-\left(n, c_{1}, c_{2}\right)$ with SSP under the conditions of IPD is evidently more efficient than the SSP under the conditions of IPD since $T N T-\left(n, c_{1}, c_{2}\right)$ requires fewer samples for inspection.

\section{Conclusion:}

The proposed $T N T-\left(n, c_{1}, c_{2}\right)$ sampling scheme is suitable when there is a possibility of intervention during the production process with aim to produce the good products. The OC function for $T N T-\left(n, c_{1}, c_{2}\right)$ under the conditions of Intervened Poisson distribution is derived and a selection of optimum $T N T-\left(n, c_{1}, c_{2}\right)$ scheme which will provide protection to both producer and consumer is discussed through an iterative procedure. Further, the efficiency of proposed $T N T-\left(n, c_{1}, c_{2}\right)$ scheme over its reference plans has been discussed and proved that the proposed $T N T-\left(n, c_{1}, c_{2}\right)$ sampling scheme is efficient over its reference plans. The proposed plan can be applied in food processing units where the food is processed through various stages and there is a possibility to have an intervention during the process. Further, the plan can also be used in various other fields like agriculture, medicine etc where the human intervention is much involved. 


\section{Reference:}

1. Calvin T W. (1977). TNT Zero Acceptance Number Sampling. American Society for Quality Control Annual Technical Conference Transactions, Philadelphia, PA, pp. 35 39.

2. Cameron J M. (1952). Tables for Constructing and for Computing the Operating Characteristics of Single Sampling Plans, Industrial Quality Control, 9(1): pp. 37 - 39.

3. Dodge H F. (1965). Evaluation of a Sampling Inspection System Having Rules for Switching Between Normal and Tightened Inspection. Technical Report No.14, The Statistics Center, Rutgers - The State University, New Jersey, US.

4. Dodge H F. (1969). Notes on the Evaluation of Acceptance Sampling Plans. Journal of Quality Technology, 1(2): pp. 77-88.

5. Huang M, Fung K Y. (1989). Intervened truncated Poisson distribution, Sankhya 51: pp. 302-310.

6. Pradeepa Veerakumari K, Azarudheen S (2016). Evaluation of Single Sampling Plan under the conditions of Intervened Poisson distribution, Communication in statistics Simulation and Computation, 46(8): pp. 6104 - 6114.

7. Schilling E G, Neubauer D V. (2009). Acceptance Sampling in Quality Control. Second Edition, CRC Press, Taylor and Francis Group, New York.

8. Schilling E G. (1982). Acceptance Sampling in Quality Control. Marcel Dekker, Inc., New York.

9. Shanmugam R. (1985). An intervened Poisson distribution and its medical application. Biometrics, 41: pp. 1025-1029.

10. Shanmugam R. (2001). Predicting a "successful" prevention of an epidemic, Communication in Statistics-Theory \& Methods, 30(1): pp. 93-103.

11. Soundararajan V, Vijayaraghavan R. (1990). Construction and Selection of Tightened Normal - Tightened (TNT) Plans. Journal of Quality Technology. 22(2): pp. 146 - 153.

12. Soundararajan V, Vijayaraghavan R. (1992). Construction and Selection of TightenedNormal-Tightened Sampling Inspection Scheme of Type TNT [ $\mathrm{n}_{1}, \mathrm{n}_{2}$; c]. Journal of Applied Statistics, 19(3): pp. 339-349.

13. Subrmani K, Haridoss V. (2012). Selection of a Tightened-Normal-Tightened systems for given values of Acceptable Quality Level and Limiting Quality Level using Weighted Poisson distribution. International Journal of Decision Sciences, Risk and Management, 4(3/4): pp. 300-313.

14. Vijayaraghavan R, Loganathan A, Rajalakshmi D. (2014). Selection of Minimum Sample Size Tightened- Normal-Tightened Sampling Inspection Schemes. Journal of Testing and Evaluation, 42(1): pp. 204-210.

15. Vijayaraghavan R, Soundararajan V. (1996). Procedures and Tables for the Selection of Tightened-Normal-Tightened TNT $\left(\mathrm{n} ; \mathrm{c}_{1}, \mathrm{c}_{2}\right)$ Sampling Schemes. Journal of Applied Statistics, 23(1): pp. 69-79. 